\title{
Dependence of the Neutron and Gamma-ray Emission of Mercury on Its Surface Composition and Temperature
}

\author{
N. A. Gun'ko', A. S. Kozyrev"*, I. G. Mitrofanov², and A. I. Tsygan' \\ ${ }^{1}$ A.F. Ioffe Physical Technical Institute, Russian Academy of Sciences, \\ Polytekhnicheskaya ul. 26, St. Petersburg, 194021 Russia \\ ${ }^{2}$ Space Research Institute, Russian Academy of Sciences, \\ Profsoyuznaya ul. 84/32, Moscow, 117997 Russia \\ Received December 29, 2010; in final form, June 15, 2011
}

\begin{abstract}
Results of computations of the energy spectrum of neutrons radiated by the planet Mercury, generated by the action of cosmic rays, are presented. The dependence of the neutron radiation on both the temperature and composition of the subsurface layers of the planetary regolith are investigated. The fluxes of $\gamma$-rays in lines of aluminum $\left({ }^{27} \mathrm{Al}, 7.7240 \mathrm{MeV}\right)$, silicon $\left({ }^{28} \mathrm{Si}, 3.5390 \mathrm{MeV}\right)$, calcium $\left({ }^{40} \mathrm{Ca}\right.$, $1.9427 \mathrm{MeV}$ ), and iron $\left({ }^{56} \mathrm{Fe}, 7.6312\right.$ and $\left.7.6456 \mathrm{MeV}\right)$ generated during neutron capture by these nuclei are also calculated. The intensity of the radiation in these lines depends on both the composition and temperature of the surface. This must be taken into account when interpreting measurements of $\gamma$-ray radiation from nuclear lines produced in neutron capture reactions on the substance of Mercury.
\end{abstract}

DOI: $10.1134 / \mathrm{S} 1063772912030031$

\section{INTRODUCTION}

Experiments on the spectra of neutrons and $\gamma$ rays emitted from the surfaces of planets and other celestial bodies without dense atmospheres carried out on spacecraft [1] have led to the need to predict the properties of this radiation as functions of the surface composition and temperature. Nuclear radiation from the surfaces of celestial bodies arises when the nuclei of elements comprising the subsurface are bombarded by cosmic rays (protons and $\alpha$ particles). The nuclei emit evaporative and cascade neutrons (and protons), which are absorbed and scattered in the matter of the planetary surface. Some of these particles escape from the planet, flying into circumplanetary space. Interactions of neutrons with nuclei can lead to inelastic scattering and neutron-capture reactions in which nuclear $\gamma$-rays are emitted, with each nucleus having its particular set of $\gamma$-ray lines. It is these neutrons and $\gamma$-rays that comprise the fluxes of nuclear radiation emerging from the surfaces of celestial bodies that are detected by neutron and $\gamma$-ray spectrometers on board spacecraft at distances from these bodies not exceeding several hundred kilometers. Analyses of such measurements can be used to determine the composition of the main rock-forming elements and hydrogen in the upper layer of the surface with a thickness of about one meter.

\footnotetext{
${ }^{*}$ E-mail: kozyrev@iki.rssi.ru
}

The Mercury Gamma-ray and Neutron Spectrometer (MGNS) is currently being developed at the Space Research Institute of the Russian Academy of Sciences, on contract to the Federal Space Agency. This instrument is intended for measurements of secondary nuclear $\gamma$-ray emission and neutrons from the surface of Mercury onboard the "BepiColombo" interplanetary spacecraft of the European Space Agency [1]. Mercury does not have an atmosphere or strong magnetic field, so that energetic charged cosmic-ray particles can freely penetrate into the upper layer of its surface, where they can generate secondary nuclear emission in the form of neutrons and $\gamma$-rays. Mercury is the closest planet to the Sun. In daytime, its surface is heated by the flux of solar radiation, with the maximum temperatures reaching $725 \mathrm{~K}$. The nightime surface faces open space, and has a temperature of $90 \mathrm{~K}$. This substantial difference between the daytime and nightime temperatures of about $600 \mathrm{~K}$ could affect the flux of super-thermal and thermal neutrons and the intensity of $\gamma$-rays in lines arising due to the capture of secondary neutrons by nuclei in the surface.

Nine scenarios for the formation of Mercury are currently known, which predict four main models for the composition of its surface material [1-5]: the Chondrite Model (ChM, Model 1), the Equilibrium Condensation Model (ECM, Model 2), the Refractory Rich Model (RRM, Model 3), and the Volatile Rich 
Table 1. Abundances of main rock-forming elements in the surface material of Mercury according to the four main models for its composition (in percent by mass)

\begin{tabular}{l|r|r|r|c}
\hline Elements & $\begin{array}{c}\text { Model 1 } \\
\text { ChM }\end{array}$ & $\begin{array}{c}\text { Model 2 } \\
\text { ECM }\end{array}$ & $\begin{array}{c}\text { Model 3 } \\
\text { RRM }\end{array}$ & $\begin{array}{c}\text { Model 4 } \\
\text { VRM }\end{array}$ \\
\hline $\mathrm{Mg}$ & 20.30 & 24.20 & 20.90 & 19.50 \\
$\mathrm{Al}$ & 3.40 & 5.10 & 8.80 & 1.70 \\
$\mathrm{Si}$ & 22.10 & 19.10 & 15.20 & 21.40 \\
$\mathrm{O}$ & 45.10 & 45.00 & 43.80 & 43.60 \\
$\mathrm{Ca}$ & 3.70 & 6.20 & 10.90 & 2.20 \\
$\mathrm{Ti}$ & 0.20 & 0.29 & 0.40 & - \\
$\mathrm{Cr}$ & 2.30 & 0.07 & - & - \\
$\mathrm{Fe}$ & 2.90 & 0.04 & - & 11.60 \\
\hline
\end{tabular}

Model (VRM, Model 4). The abundances of the main rock-forming elements for these models are given in Table 1 . The task of space-based $\gamma$-ray spectroscopy of Mercury is estimation of the abundances of the main elements in its surface material, and using this information to select the most probable model for the formation of this planet [1]. This selection will be based on the relative intensities of several main $\gamma$-ray lines, for which the models for the formation of Mercury predict different values (Table 1).

As an example, let us consider the $7.72 \mathrm{MeV}$ ${ }^{27} \mathrm{Al}$ and $3.54 \mathrm{MeV}{ }^{28} \mathrm{Si}$ lines, which are generated in neutron capture reactions. The intensity of the $7.72 \mathrm{MeV} \mathrm{Al}$ line can vary by a factor of five for the various models for the formation of Mercury (Table 1 ). The intensity of the $3.54 \mathrm{MeV}$ Si line should also vary between models, but much less, within $30 \%$ ( Table 1). Below, we use the flux in the Si line for model ChM (Model 1), which has the maximum Si abundance, as a reference for our investigation of the effect of the composition and temperature of the surface material.

It is obvious that trustworthy estimates of the composition of the Mercurian surface material will require a reliable translation from measurements of the $\gamma$-ray line intensities formed in neutron capture reactions to abundance estimates for the corresponding elements. This translation must take into account the temperature dependence of the mean reaction cross section. The aim of our current study is to investigate the influence of the composition and temperature on the formation and transport of neutrons and $\gamma$-rays in the indicated lines. We used the four main composition models to calculate the expected neutron and $\gamma$-ray fluxes (Table 1 ). We investigated the temperature dependence for subsurface temperatures from 90 to $925 \mathrm{~K}$, corresponding to the conditions for night and day on the surface of Mercury.

\section{MODELING OF THE NEUTRON SPECTRA}

We calculated the flux of neutrons using MonteCarlo simulations. The computational process was based on simulations of random quantities - the parameters of the processes leading to the creation and propagation of neutrons in a given medium, taking into account the formation and propagation of $\gamma$-rays. The set of parameters included the initial characteristics of the neutron emitted, the mean free path, the scattering angle, parameters characterizing the interaction of the neutron with nuclei (and the set of nuclei with which these interactions occur), and the energy and spatial coordinates of the scattered neutron. These computations yielded estimates of the neutron fluxes from the surface of Mercury for various energy ranges.

The direct simulations of the source of the neutrons in the depth of the subsurface layer required substantial amounts of computer time. Therefore, we applied a stacking approach, taking into account weighting coefficients. The medium was divided into thin layers with different depths, in which the initial neutrons were emitted with random initial parameters (energy, depth, and direction of flight), according to the adopted distribution functions for these parameters (see Appendix A). The deepest layer in a given computation was determined from the condition that the number of neutrons emerging from the surface be less than $0.1 \%$ of the total number of neutrons emitted. In this way, we cut out inefficient versions of the computations, corresponding to neutrons randomly wandering in deep layers. When computing the neutron spectra, the energy interval was divided into sections that were equal on a logarithmic scale, whose number depended on the temperature, and reached of the order of 200. The accuracy of the derived characteristics (neutron and $\gamma$-ray fluxes) were estimated in the computations; the estimated uncertainties did not exceed $0.3 \%$. For neutron energies close to $k T / 10$, the uncertainties in the computed neutron energy spectra reached several percent. The total number of neutrons participating in the simulations varied from five to eight million. The computations were carried out for four subsurface temperatures: 90, 300, 500, and $725 \mathrm{~K}$ (see Appendix A).

Note that all the neutron fluxes were calculated per primary neutron formed in the subsurface material. This approach enables us to analyze variations in the spectral energy distribution for the emerging neutrons as a function of the composition and temperature of the surface material, but not to estimate variations in the absolute flux of emerging neutrons as 


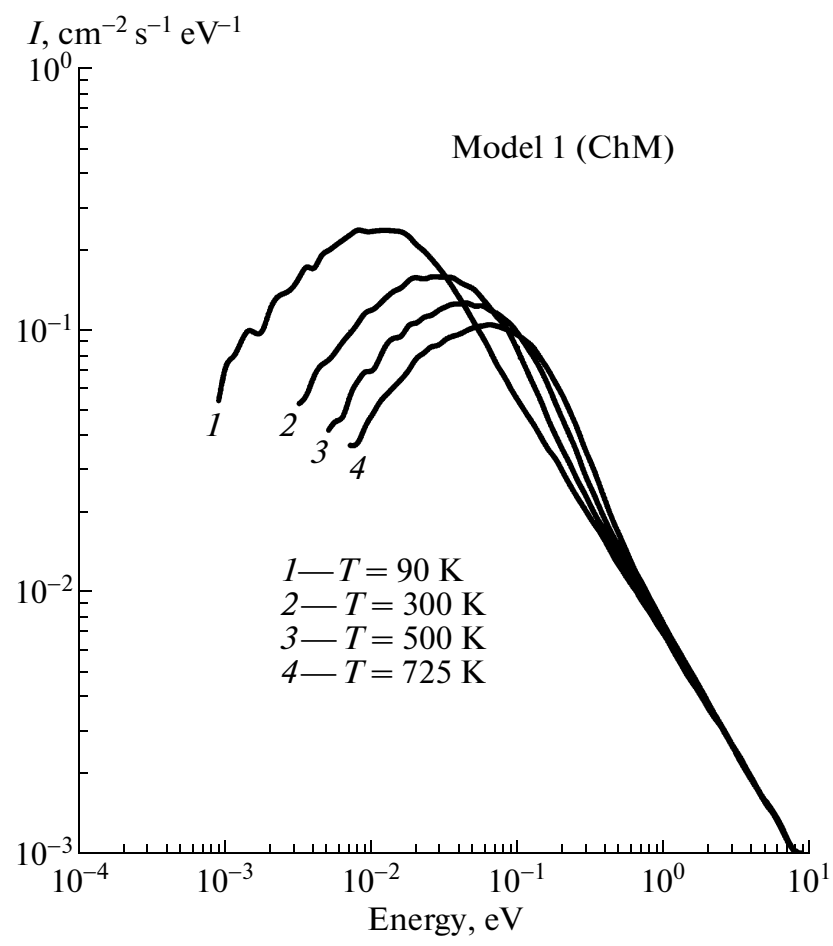

Fig. 1. Spectral flux density of neutrons (with energies less than $10 \mathrm{eV}$ ) for various surface temperatures of Mercury and regolith model ChM as a function of neutron energy. The neutron fluxes have been normalized to represent the fluxes per neutron formed in the subsurface.

a function of the surface composition. It is known that the number of primary neutrons created per energetic charged cosmic-ray particle varies with the mean nuclear charge $\langle Z\rangle$. However, this effect does not depend on the temperature of the material, and so is not considered here. On the other hand, the temperature influences the deceleration and thermalization of the primary neutrons, so that the flux of emerging neutrons with various energies can be considered per primary neutron. Figures $1-4$ present the calculated neutron energy spectra for energies $<10 \mathrm{eV}$ separately for each model.

The computations show that our estimates of the number of neutrons emerging from the surface of Mercury as a fraction of the number of primary neutrons depends weakly on the surface composition and temperature. Table 2 presents the total fluxes of neutrons emerging from the surface (in $\mathrm{cm}^{-2} \mathrm{~s}^{-1}$ ) at energies of $k T / 10-10^{7} \mathrm{eV}$ per neutron emitted from $1 \mathrm{~cm}^{2}$ of the surface per $1 \mathrm{~s}$ (also in $\mathrm{cm}^{-2} \mathrm{~s}^{-1}$ ). The characteristic total emergent flux is $0.28-0.29$ per emitted neutron, with the variations for the different composition models being $2-3 \%$ and the variations for different temperatures being $1-2 \%$.

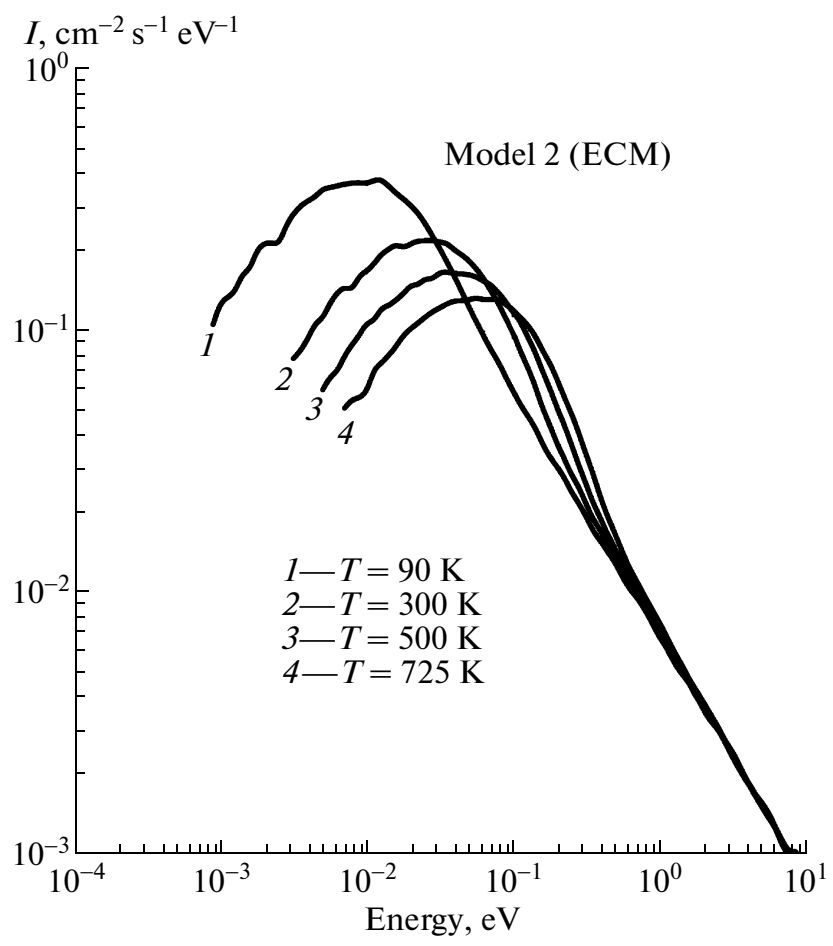

Fig. 2. Same as Fig. 1 for regolith model ECM.

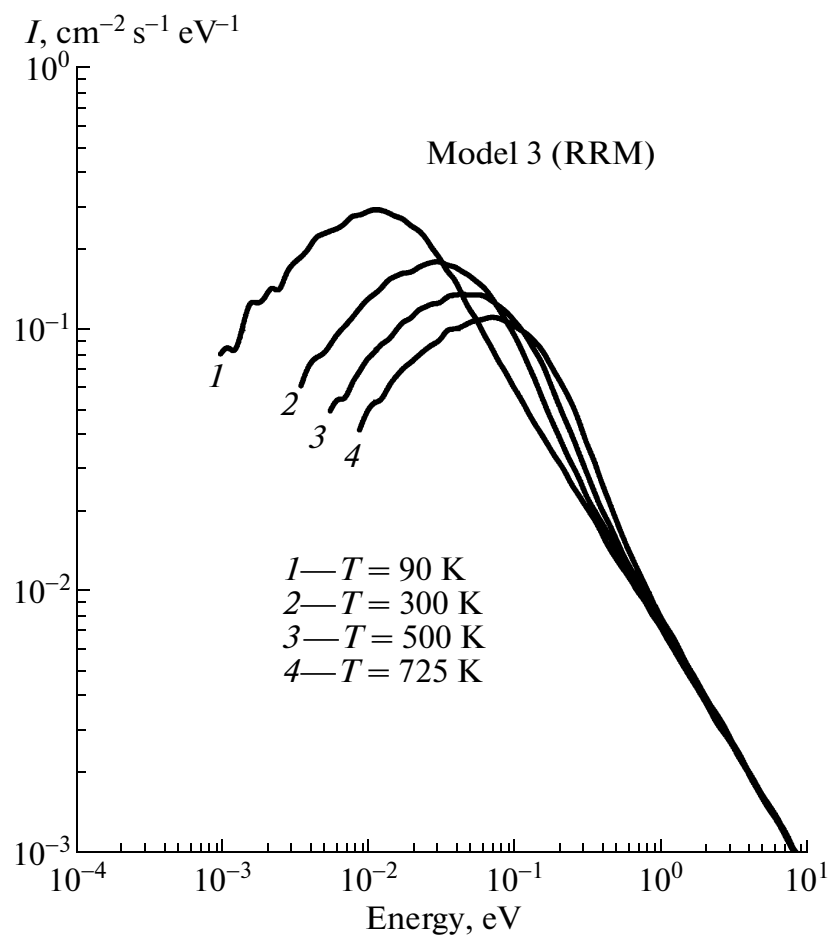

Fig. 3. Same as Fig. 1 for regolith model RRM. 
Table 2. Estimates of the total flux of secondary neutrons from the surface (in $\mathrm{cm}^{-2} \mathrm{~s}^{-1}$ ) as a function of the surface composition and mean temperature (per neutron emitted)

\begin{tabular}{c|c|c|c|c}
\hline \multirow{2}{*}{ Model } & \multicolumn{4}{|c}{ Surface temperature, K } \\
\cline { 2 - 5 } & 90 & 300 & 500 & 725 \\
\hline $1($ ChM $)$ & 0.281 & 0.284 & 0.285 & 0.286 \\
$2($ ECM $)$ & 0.282 & 0.285 & 0.286 & 0.288 \\
$3($ RRM $)$ & 0.276 & 0.278 & 0.279 & 0.281 \\
$4($ VRM $)$ & 0.275 & 0.276 & 0.277 & 0.278 \\
\hline
\end{tabular}

Table 3. Estimates of the flux of secondary neutrons with various energies (in $\mathrm{cm}^{-2} \mathrm{~s}^{-1}$ ) as a function of the surface composition and mean temperature (per neutron emitted). (Quantities in parantheses show the fractions of the total flux comprised by the fluxes of thermal, epithermal, and fast neutrons in percent)

\begin{tabular}{c|c|c|c|c|c|c}
\hline \multirow{3}{*}{ Model } & \multicolumn{5}{|c|}{ Surface temperature 90 K } & \multicolumn{3}{c}{ Surface temperature 725 K } \\
\cline { 2 - 7 } & \multicolumn{7}{|c}{ Neutron energy, eV } \\
\cline { 2 - 7 } & $<0.5$ & $0.5-100$ & $>100$ & $<0.5$ & $0.5-100$ & $>100$ \\
\hline \multirow{2}{*}{$1(\mathrm{ChM})$} & $2.14 \times 10^{-2}$ & $3.73 \times 10^{-2}$ & $2.23 \times 10^{-1}$ & $2.48 \times 10^{-2}$ & $3.85 \times 10^{-2}$ & $2.23 \times 10^{-1}$ \\
& $(7.6)$ & $(13.2)$ & $(79.2)$ & $(8.7)$ & $(13.4)$ & $(77.9)$ \\
$2(\mathrm{ECM})$ & $2.59 \times 10^{-2}$ & $3.81 \times 10-2$ & $2.18 \times 10^{-1}$ & $3.00 \times 10^{-2}$ & $3.93 \times 10^{-2}$ & $2.18 \times 10^{-1}$ \\
& $(9.2)$ & $(13.5)$ & $(77.3)$ & $(10.4)$ & $(13.7)$ & $(75.9)$ \\
$3(\mathrm{RRM})$ & $2.26 \times 10^{-2}$ & $3.74 \times 10^{-2}$ & $2.16 \times 10^{-1}$ & $2.63 \times 10^{-2}$ & $3.86 \times 10^{-2}$ & $2.16 \times 10^{-1}$ \\
& $(8.2)$ & $(13.5)$ & $(78.3)$ & $(9.4)$ & $(13.7)$ & $(76.9)$ \\
$4(\mathrm{VRM})$ & $1.46 \times 10^{-2}$ & $3.43 \times 10^{-2}$ & $2.26 \times 10^{-1}$ & $1.66 \times 10^{-2}$ & $3.53 \times 10^{-2}$ & $2.26 \times 10^{-1}$ \\
& $(5.3)$ & $(12.5)$ & $(82.2)$ & $(6.0)$ & $(12.7)$ & $(81.3)$ \\
\hline
\end{tabular}

Table 3 presents the neutron fluxes (in $\mathrm{cm}^{-2} \mathrm{~s}^{-1}$, per neutron emitted) for energies $k T / 10-0.5 \mathrm{eV}, 0.5-$ $100 \mathrm{eV}$, and $>100 \mathrm{eV}$. These fluxes correspond to thermal, epithermal, and fast neutrons. The fluxes of fast neutrons do not depend on temperature: their energy is high compared to the thermal energy of the nuclei with which they interact. The fluxes of thermal neutrons grow appreciably with increasing temperature. This is due to the fact that the mean

Table 4. Ratio of fluxes of thermal and fast neutrons $(\xi)$ for extreme values of the surface temperature

\begin{tabular}{c|c|c}
\hline \multirow{2}{*}{ Model } & \multicolumn{2}{|c}{ Surface temperature } \\
\cline { 2 - 3 } & $\begin{array}{c}\text { minimum } \\
(90 \mathrm{~K})\end{array}$ & $\begin{array}{c}\text { maximum } \\
(725 \mathrm{~K})\end{array}$ \\
\hline 1 (ChM) & 0.096 & 0.112 \\
$2(\mathrm{ECM})$ & 0.119 & 0.137 \\
$3(\mathrm{R} M)$ & 0.105 & 0.122 \\
$4(\mathrm{VRM})$ & 0.064 & 0.074 \\
\hline
\end{tabular}

relative velocity of the nuclei and neutrons increases as the temperature increases, which reduces the cross section for neutron capture by the nuclei. The modest increase of the flux of epithermal neutrons with increasing temperature is likewise due to variations in the flux of thermal particles-the computed distributions of the flux of emerging neutrons (Figs. 1-4) clearly show that, as the temperature grows, some of the thermalized particles acquire energies higher than $0.5 \mathrm{eV}$ and "penetrate" into the epithermal range, $0.5-100 \mathrm{eV}$.

Table 3 also gives ratios of the fluxes of thermal, epithermal, and fast neutrons to the total flux (quantities in parantheses). These ratios characterize the energy spectrum of the generated neutrons or, alternatively, the efficiency of the deceleration of the neutrons in the subsurface. Therefore, they reflect the dependence of the spectrum of emerging neutrons on the temperature of the surface material. Note that these flux ratios in various spectral intervals do not depend on whether the computations of the flux of emerging neutrons are renormalized per primary neutron or per energetic charged cosmic-ray particle. 
Table 5. Estimated radiation fluxes in the $\mathrm{Si} 3.54 \mathrm{MeV}$ line (in $\mathrm{cm}^{-2} \mathrm{~s}^{-1}$ ) as a function of the surface composition and temperature (per neutron emitted). (Quantities in square brackets in the last column show ratios of the fluxes for extreme values of the surface temperature)

\begin{tabular}{c|c|c|c|c|c}
\hline \multirow{2}{*}{ Model } & $\begin{array}{c}\text { Si } \\
\text { abundance, }\end{array}$ & \multicolumn{4}{|c}{ Temperature, $\mathrm{K}$} \\
\cline { 3 - 5 } & $\%$ & 90 & 300 & 500 & 725 \\
\hline \multirow{2}{*}{$1(\mathrm{ChM})$} & 22.1 & $4.46 \times 10^{-3}$ & $4.23 \times 10^{-3}$ & $4.09 \times 10^{-3}$ & $3.99 \times 10^{-3}[0.89]$ \\
& $(1.0)$ & $(1.0)$ & $(1.0)$ & $(1.0)$ & $(1.0)$ \\
$2(\mathrm{ECM})$ & 19.1 & $4.36 \times 10^{-3}$ & $4.03 \times 10^{-3}$ & $3.83 \times 10^{-3}$ & $3.67 \times 10^{-3}[0.84]$ \\
& $(0.86)$ & $(0.98)$ & $(0.95)$ & $(0.94)$ & $(0.92)$ \\
$3(\mathrm{RRM})$ & 15.2 & $3.03 \times 10^{-3}$ & $2.95 \times 10^{-3}$ & $2.85 \times 10^{-3}$ & $2.75 \times 10^{-3}[0.91]$ \\
& $(0.69)$ & $(0.68)$ & $(0.70)$ & $(0.70)$ & $(0.69)$ \\
$4(\mathrm{VRM})$ & 21.4 & $3.76 \times 10^{-3}$ & $3.69 \times 10^{-3}$ & $3.62 \times 10^{-3}$ & $3.59 \times 10^{-3}[0.95]$ \\
& $(0.97)$ & $(0.84)$ & $(0.87)$ & $(0.89)$ & $(0.90)$ \\
\hline
\end{tabular}

The relative quantities in parantheses correspond to the fraction of the values for Model ChM.

This means that the flux ratios presented in Table 3 can be directly compared with observations.

It follows from the computations ( Table 3 ) that the relative flux of epithermal neutrons as a fraction of the total flux is essentially independent of both the composition and the temperature of the surface material: for all four models, this fraction is $12.5-13.5 \%$ for a surface temperature of $90 \mathrm{~K}$ and $12.7-13.7 \%$ for $725 \mathrm{~K}$. On the other hand, the ratio $\xi$ of the fluxes of thermal and fast neutrons is a useful physical quantity that displays a fairly strong dependence on both the composition and temperature (Table 4).

The computed values of $\xi$ vary by nearly a factor of two between the maximum values obtained, for the ECM (Model 2; 12-14\%), and the minimum values, for the VRM (Model 4; 6-7\%) (Table 4). The decrease in the flux of thermal neutrons for the VRM (Model 4) is due to the high mass fraction of iron (Table 1), and the fact that iron nuclei are efficient $a b-$ sorbers of thermal neutrons. For all four models, the fraction of thermal neutrons grows with temperature, and the parameter $\xi$ increases by roughly a factor of $1.15-1.17$ (i.e., by $15-17 \%$ ).

Thus, based on numerical simulations of the neutron energy spectra, we can conclude that the daily variations of the thermal flux relative to the fastneutron flux comprise $15-17 \%$, essentially independent of the composition, with the relative flux of thermal neutrons as a fraction of the total flux varying by nearly a factor of two. Therefore, when studying the composition of the surface material based on measurements of variations in the flux of thermal neutrons, it is necessary to correct the observational data for the temperature variations of the thermal neutrons. Moreover, temperature effects must be taken into account when interpreting measurements of $\gamma$-ray lines emitted during neutron-capture reactions, which depend on both the number of incident thermal neutrons and the relative velocity of their collisions with the capturing nuclei. The following section of this paper is dedicated to this question.

\section{MODELING OF THE FLUXES IN $\gamma$-RAY LINES EMITTED}

\section{DURING NEUTRON CAPTURE REACTIONS}

We used our computations of the flux of neutrons from the surface of Mercury to investigate the influence of the temperature of the subsurface on the intensity of nuclear $\gamma$-ray line emission generated in neutron-capture reactions. It is obvious that emission in $\gamma$-ray lines generated in inelastic-scattering reactions should not be subject to temperature effects, since such interactions take place in collisions with fast neutrons. When computing the $\gamma$-ray flux from the surface, we considered separately neutron capture by nuclei of ${ }^{40} \mathrm{Ca},{ }^{28} \mathrm{Si},{ }^{27} \mathrm{Al}$, and ${ }^{56} \mathrm{Fe}$, which generate lines at 1.94, 3.54, 7.72, and $(7.63+7.65) \mathrm{MeV}$, respectively. The partial probability for the emission of these lines during neutron capture by these nuclei is presented in Appendix B, together with the elemental composition. During the propagation of $\gamma$-rays in nuclear lines in matter, the main process attenuating the line intensity is Compton scattering. In addition, $\gamma$-rays will be absorbed during the creation of electron-positron pairs. Both these processes were taken into account when computing the fluxes in these lines.

Table 5 and Fig. 5 present the $\gamma$-ray flux (in units of $\mathrm{cm}^{-2} \mathrm{~s}^{-1}$ ) in the $3.54 \mathrm{MeV}$ nuclear line of Si calculated per neutron emitted per $\mathrm{cm}^{2}$ of the surface per second. The Si abundance varies only insignificantly between the various models for the surface material 


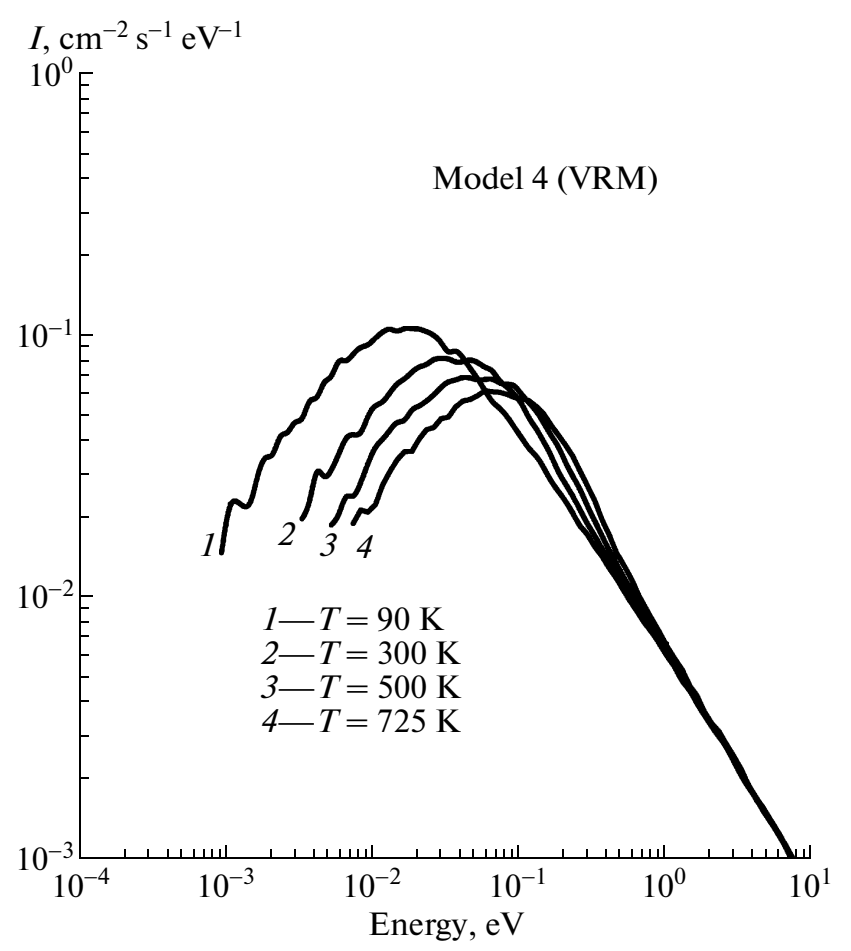

Fig. 4. Same as Fig. 1 for regolith model VRM.

(Table 1), and the variation in the intensity of this line between the various models is likewise modest, within $30 \%$. This line can thus be used as a reference feature for studies of the influence of the composition and temperature of the surface material on the flux of thermal neutrons. Table 8 (column 2) presents the absolute and relative abundances of Si [the latter as a fraction of the value $22.1 \%$ for the ChM (Model 1)].

A comparison of the ratio of the fluxes in the $3.54 \mathrm{MeV}$ line and the relative abundance of $\mathrm{Si}$ indicates that the relative fluxes for a temperature of $90 \mathrm{~K}$ for the ECM (Model 2) and the VRM (Model 4), which have Si abundances of 0.86 and 0.97 of the maximum value for Model 1 , respectively, display the opposite relation, i.e., the fluxes comprise 0.98 and 0.84 of the maximum value for Model 1 , respectively. This "inversion" of the relations for the abundance and line fluxes for Models 2 and 4 is due to the fact that the thermal-neutron fluxes for these two models differ appreciably. The parameter $\xi$ is equal to its maximum, 0.119 , and minimum, 0.064 , values for Models 2 and 4, respectively. The relatively high flux of thermal neutrons for Model 2 leads to an enhancement in the flux in this nuclear line compared to its flux for Model 1, while the low flux of thermal neutrons for Model 4 leads to appreciable attenuation of the line flux.

The thermal-neutron flux for Model 3 essentially coincides with the flux for Model $1(\xi=0.1)$, so that

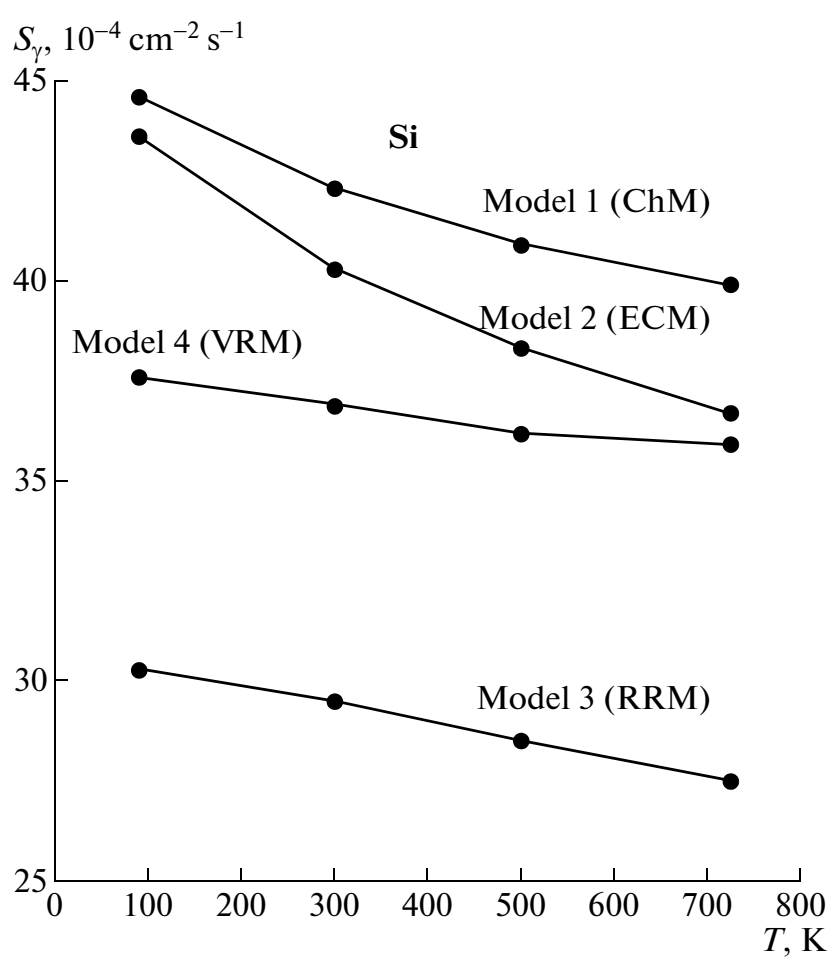

Fig. 5. Results of modeling of the intensity of the Si 3.54$\mathrm{MeV} \gamma$-ray line as a function of the surface temperature of Mercury, for the various models of the regolith composition (the fluxes are given per neutron emitted per $\mathrm{cm}^{2}$ of the surface per second).

the relative fluxes in the $3.54 \mathrm{MeV}$ line for these two models agree well with their relative $\mathrm{Si}$ abundances.

The intensity of the $3.54 \mathrm{MeV}$ line is reduced when the surface temperature is raised from 90 to $725 \mathrm{~K}$ (Table 5, Fig. 5). This attenuation of the line flux with increasing temperature from 90 to $725 \mathrm{~K}$ is presented in square brackets in the last column of Table 8 , as the ratio of the fluxes for the highest and lowest temperatures. This effect is maximum (16\%) for the composition in the ECM (Model 2), since the fraction of thermal neutrons in the total neutron flux is maximum for this model, and precisely reactions with thermal neutrons give rise to the temperaturerelated reduction of the $\gamma$-rays created by neutroncapture reactions. With increasing temperature, the weakening of the flux due to the reduction in the reaction cross section dominates over the increase in the number of interactions due to the growth in the fraction of thermal neutrons in the incident flux (from 9.2 to $10.4 \%$; Table 3 ). It is interesting that this temperature effect overall reduces the discrepancy between the relative abundances of $\mathrm{Si}$ and the relative $3.54 \mathrm{MeV}$ line fluxes for the various models at high temperatures (see the values in parantheses in the last 
Table 6. Estimate of the flux in the Ca $1.94 \mathrm{MeV}$ line (in $\mathrm{cm}^{-2} \mathrm{~s}^{-1}$, per emitted neutron) as a function of the surface composition and temperature. (The flux ratio for the maximum and minimum temperatures are presented in square brackets in the last column)

\begin{tabular}{c|c|c|c|c|c}
\hline \multirow{2}{*}{ Model } & $\begin{array}{c}\text { Ca } \\
\text { abundance, } \\
\%\end{array}$ & \multicolumn{4}{|c}{ Temperature, $\mathrm{K}$} \\
\cline { 3 - 5 } & $\%$ & 90 & 300 & 500 & 725 \\
\hline \multirow{2}{*}{$1(\mathrm{ChM})$} & 3.7 & $1.64 \times 10^{-3}$ & $1.58 \times 10^{-3}$ & $1.54 \times 10^{-3}$ & $1.52 \times 10^{-3}[0.93]$ \\
& $(0.34)$ & $(0.35)$ & $(0.34)$ & $(0.35)$ & $(0.35)$ \\
$2(\mathrm{ECM})$ & 6.2 & $3.06 \times 10^{-3}$ & $2.75 \times 10^{-3}$ & $2.65 \times 10^{-3}$ & $2.60 \times 10^{-3}[0.85]$ \\
& $(0.57)$ & $(0.65)$ & $(0.60)$ & $(0.59)$ & $(0.59)$ \\
$3(\mathrm{RRM})$ & 10.9 & $4.73 \times 10^{-3}$ & $4.58 \times 10^{-3}$ & $4.46 \times 10^{-3}$ & $4.39 \times 10^{-3}[0.93]$ \\
& $(1.0)$ & $(1.0)$ & $(1.0)$ & $(1.0)$ & $(1.0)$ \\
$4(\mathrm{VRM})$ & 2.2 & $9.25 \times 10^{-4}$ & $9.12 \times 10^{-4}$ & $8.94 \times 10^{-4}$ & $8.98 \times 10^{-4}[0.97]$ \\
& $(0.20)$ & $(0.20)$ & $(0.20)$ & $(0.20)$ & $(0.20)$ \\
\hline
\end{tabular}

The relative quantities in parantheses correspond to the fraction of the value for the RRM.

Table 7. Estimate of the flux in the $\mathrm{Al} 7.72 \mathrm{MeV}$ line (in $\mathrm{cm}^{-2} \mathrm{~s}^{-1}$, per emitted neutron) as a function of the surface composition and temperature. (The flux ratio for the maximum and minimum temperatures are presented in square brackets in the last column)

\begin{tabular}{|c|c|c|c|c|c|}
\hline \multirow{2}{*}{ Model } & \multirow{2}{*}{$\begin{array}{c}\mathrm{Al} \\
\text { abundance, } \\
\% \\
\end{array}$} & \multicolumn{4}{|c|}{ Temperature, K } \\
\hline & & 90 & 300 & 500 & 725 \\
\hline $1(\mathrm{ChM})$ & $\begin{array}{c}3.4 \\
(0.39)\end{array}$ & $\begin{array}{c}4.28 \times 10^{-4} \\
(0.37)\end{array}$ & $\begin{array}{l}3.92 \times 10^{-4} \\
(0.38)\end{array}$ & $\begin{array}{c}3.75 \times 10^{-4} \\
(0.38)\end{array}$ & $\begin{array}{c}3.55 \times 10^{-4}[0.83] \\
(0.38)\end{array}$ \\
\hline $2(\mathrm{ECM})$ & $\begin{array}{c}5.1 \\
(0.58)\end{array}$ & $\begin{array}{l}7.73 \times 10^{-4} \\
(0.67)\end{array}$ & $\begin{array}{l}6.81 \times 10^{-4} \\
\quad(0.65)\end{array}$ & $\begin{array}{l}6.29 \times 10^{-4} \\
\quad(0.65)\end{array}$ & $\begin{array}{c}5.94 \times 10^{-4}[0.77] \\
(0.64)\end{array}$ \\
\hline $3(\mathrm{RR} M)$ & $\begin{array}{r}8.8 \\
(1.0)\end{array}$ & $\begin{array}{c}1.15 \times 10^{-3} \\
(1.0)\end{array}$ & $\begin{array}{c}1.04 \times 10^{-3} \\
(1.0)\end{array}$ & $\begin{array}{l}9.75 \times 10^{-4} \\
\quad(1.0)\end{array}$ & $\begin{array}{c}9.29 \times 10^{-4}[0.81] \\
(1.0)\end{array}$ \\
\hline $4(\mathrm{VRM})$ & $\begin{array}{c}1.7 \\
(0.19)\end{array}$ & $\begin{array}{c}1.65 \times 10^{-4} \\
(0.14)\end{array}$ & $\begin{array}{c}1.58 \times 10^{-4} \\
(0.15)\end{array}$ & $\begin{array}{l}1.52 \times 10^{-4} \\
(0.16)\end{array}$ & $\begin{array}{c}1.47 \times 10^{-4}[0.89] \\
(0.16)\end{array}$ \\
\hline
\end{tabular}

The relative quantities in parantheses correspond to the fraction of the value for the RRM.

Table 8. Estimate of the flux in the Fe $7.63 \mathrm{MeV}+7.65 \mathrm{MeV}$ doublet (in $\mathrm{cm}^{-2} \mathrm{~s}^{-1}$, per emitted neutron) as a function of the surface composition and temperature. (The flux ratio for the maximum and minimum temperatures are presented in square brackets in the last column)

\begin{tabular}{|c|c|c|c|c|c|}
\hline \multirow{2}{*}{ Model } & \multirow{2}{*}{$\begin{array}{c}\mathrm{Fe} \\
\text { abundance, } \\
\%\end{array}$} & \multicolumn{4}{|c|}{ Temperature, K } \\
\hline & & 90 & 300 & 500 & 725 \\
\hline $1(\mathrm{ChM})$ & $\begin{array}{c}2.9 \\
(0.25)\end{array}$ & $\begin{array}{l}2.48 \times 10^{-3} \\
\quad(0.38)\end{array}$ & $\begin{array}{l}2.21 \times 10^{-3} \\
\quad(0.35)\end{array}$ & $\begin{array}{l}2.06 \times 10^{-3} \\
(0.34)\end{array}$ & $\begin{array}{c}1.94 \times 10^{-3}[0.78] \\
(0.34)\end{array}$ \\
\hline $2(\mathrm{ECM})$ & $\begin{array}{c}0.04 \\
(0.003)\end{array}$ & $\begin{array}{l}4.30 \times 10^{-5} \\
\quad(0.007)\end{array}$ & $\begin{array}{l}3.46 \times 10^{-5} \\
(0.006)\end{array}$ & $\begin{array}{l}3.30 \times 10^{-5} \\
(0.006)\end{array}$ & $\begin{array}{c}3.06 \times 10^{-5}[0.72] \\
(0.005)\end{array}$ \\
\hline $4(\mathrm{VRM})$ & $\begin{array}{c}11.6 \\
(1.0)\end{array}$ & $\begin{array}{l}6.58 \times 10^{-3} \\
(1.0)\end{array}$ & $\begin{array}{l}6.23 \times 10^{-3} \\
\quad(1.0)\end{array}$ & $\begin{array}{l}5.98 \times 10^{-3} \\
(1.0)\end{array}$ & $\begin{array}{c}5.72 \times 10^{-3}[0.87] \\
(1.0)\end{array}$ \\
\hline
\end{tabular}

The relative quantities in parantheses correspond to the fraction of the value for the VRM. 
Table 9. Dependence of the temperature coefficient $K$ on the relative fraction of thermal neutrons $\xi$ and the $\gamma$-ray energy

\begin{tabular}{c|c|c|c|c|c}
\hline \multirow{2}{*}{ Model } & \multirow{2}{*}{$\xi, \%$} & \multicolumn{4}{|c}{ Energy of the $\gamma$-ray line, $\mathrm{MeV}$} \\
\cline { 3 - 6 } & & $1.94(\mathrm{Ca})$ & $3.54(\mathrm{Si})$ & $7.64(\mathrm{Fe})$ & $7.72(\mathrm{Al})$ \\
\hline $1(\mathrm{ChM})$ & 9.6 & 0.93 & 0.89 & 0.78 & 0.83 \\
$2(\mathrm{ECM})$ & 11.9 & 0.85 & 0.84 & 0.72 & 0.77 \\
$3(\mathrm{RRM})$ & 10.5 & 0.93 & 0.91 & - & 0.81 \\
$4(\mathrm{VRM})$ & 6.4 & 0.97 & 0.95 & 0.87 & 0.89 \\
\hline
\end{tabular}

column of Table 3 for $725 \mathrm{~K})$, compared to the lowest temperature of $90 \mathrm{~K}$.

Tables $6-8$ and Figs. $6-8$ present the calculated $\gamma$-ray fluxes (in $\mathrm{cm}^{-2} \mathrm{~s}^{-1}$ ) in the $1.94 \mathrm{MeV} \mathrm{Ca}$, $7.72 \mathrm{MeV} \mathrm{Al}$, and $7.63+7.65 \mathrm{MeV}$ nuclear lines (per neutron emitted per $\mathrm{cm}^{2}$ per second). The appreciable differences in the intensities of these lines for the various models reflects the large difference in the abundances of these nuclei in the models (Table 1).

The reduction in the line flux with growth in temperature is a somewhat unusual property of nuclear lines. The magnitude of this effect can be quantitatively characterized by the temperature coefficient $K$, equal to the ratio of the $\gamma$-ray line fluxes at high $(725 \mathrm{~K})$ and low $(90 \mathrm{~K})$ temperatures. The values of $K$

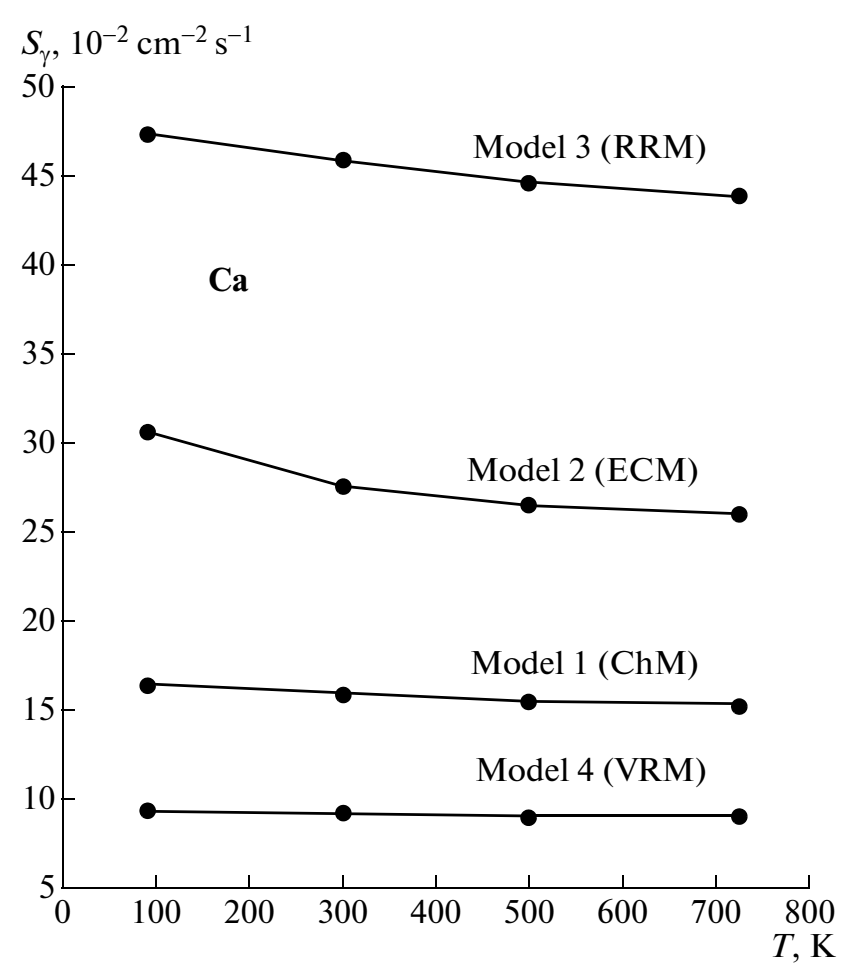

Fig. 6. Same as Fig. 5 for the $\mathrm{Ca} 1.94 \mathrm{MeV} \gamma$-ray line. for the $\gamma$-ray lines of $\mathrm{Si}, \mathrm{Ca}, \mathrm{Al}$, and Fe are presented in square brackets in the last column of Tables 6-8. Table 9 collects the $K$ values ordered as functions of both the relative flux of thermal neutrons $\xi$ (different rows) and the energy of the nuclear lines $E$ (different columns). This table shows that $K$ is lowest ( $K=$ 0.7-0.85) for high thermal-neutron fluxes, and its value approaches unity (no reduction in the line flux) as the fractional thermal-neutron flux is decreased. This can be understood if the capture of thermal neutron depends appreciably on the temperature. Another property of the temperature coefficient $K$ is that, for a fixed thermal-neutron flux, $K$ increases with the $\gamma$-ray energy: comparison of $K$ for the Ca $1.94 \mathrm{MeV}$ line and the $\mathrm{Fe}$ and $\mathrm{Al}$ lines indicates appreciable weakening of the latter lines (Table 9).

\section{DISCUSSION}

The results of our numerical simulations show that the fluxes in nuclear $\gamma$-ray lines generated by neutron-capture reactions decreases with increasing subsurface temperature. This is due to the fact that the capture cross section depends on the relative velocity of the neutron and nucleus, and increasing the temperature appreciably reduces this cross section for thermal neutrons. We expect that the reduction in the capture cross section with increasing temperature is partially canceled out by the accompanying growth in the flux of thermal and epithermal neutrons. Therefore, the weakening in the intensity of lines emitted from a heated subsurface is smaller than is implied purely by the reduction in the relative velocity of the interacting particles.

The temperature effect is about $10-30 \%$ for the $7.72-\mathrm{MeV}$ line of $\mathrm{Al}$ and the $7.63+7.65 \mathrm{MeV}$ doublet of $\mathrm{Fe}$. It is obvious that neglecting this effect could lead to substantial errors in estimates of the abundances of these elements in the regolith substance derived using data from nuclear-physical measurements. The analogous temperature effects for the 3.54 Mev line of $\mathrm{Si}$ and the $1.94 \mathrm{MeV}$ line of $\mathrm{Ca}$ are only $3-16 \%$. In some cases, the magnitude of the temperature effect is comparable to differences in 


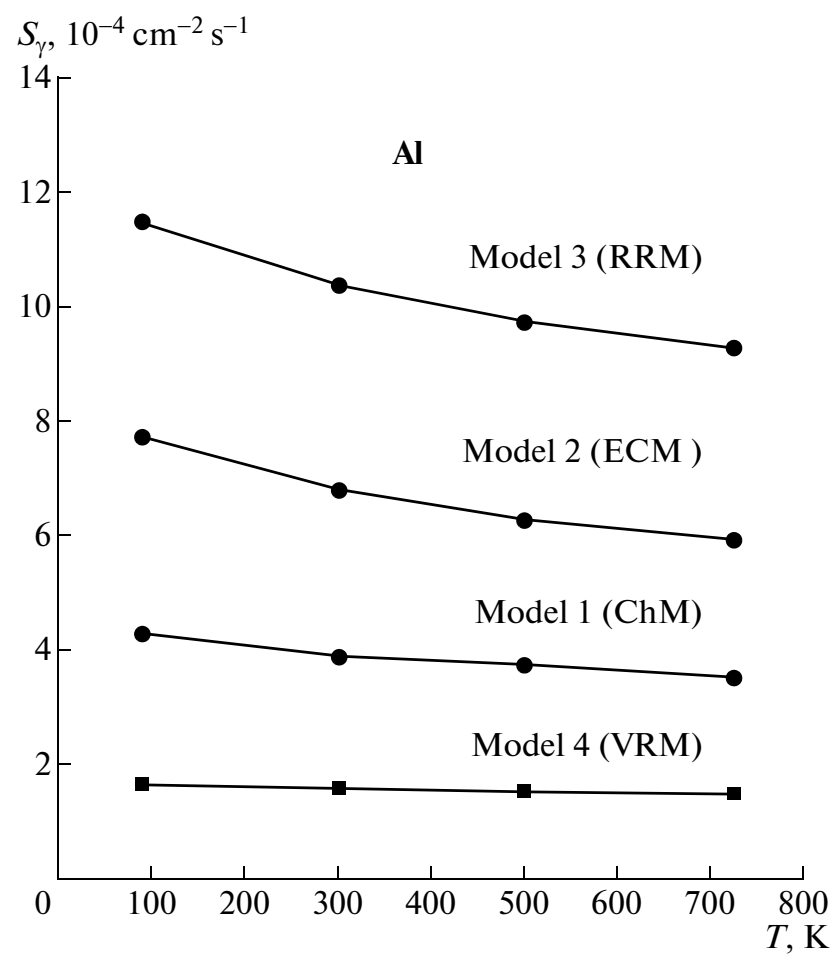

Fig. 7. Same as Fig. 5 for the $\mathrm{Al} 7.72-\mathrm{MeV} \gamma$-ray line.

the abundance estimates corresponding to the different models for the surface composition of Mercury (Table 1). Therefore, the temperature effect must be taken into account if we wish to reliably select a preferred model for the composition of the planetary surface based on measurements of nuclear lines generated by neutron-capture reactions.

The maximum temperature difference on the surface of the Moon is about $300 \mathrm{~K}$, roughly half that observed on Mercury. Accordingly, temperature variations in nuclear lines emitted by the Moon should also be about a factor of two weaker, so that the temperature effect must be taken into account for the Moon only when surface composition estimates with high accuracies of several percent are required.

Note that nuclear lines that arise during inelastic scattering of fast neutrons by nuclear are not subject to the temperature effect. The presence for a particular nucleus of lines generated via inelastic scattering and neutron capture would make it possible to use the temperature effect for the neutron capture lines to estimate the mean temperature of the subsurface layers of the planet with a thickness of several tens of centimeters. The inelastic scattering lines can be used as constant reference sources whose intensity depends only on the abundance of the relevant nucleus, and daily variations in the nuclear lines from

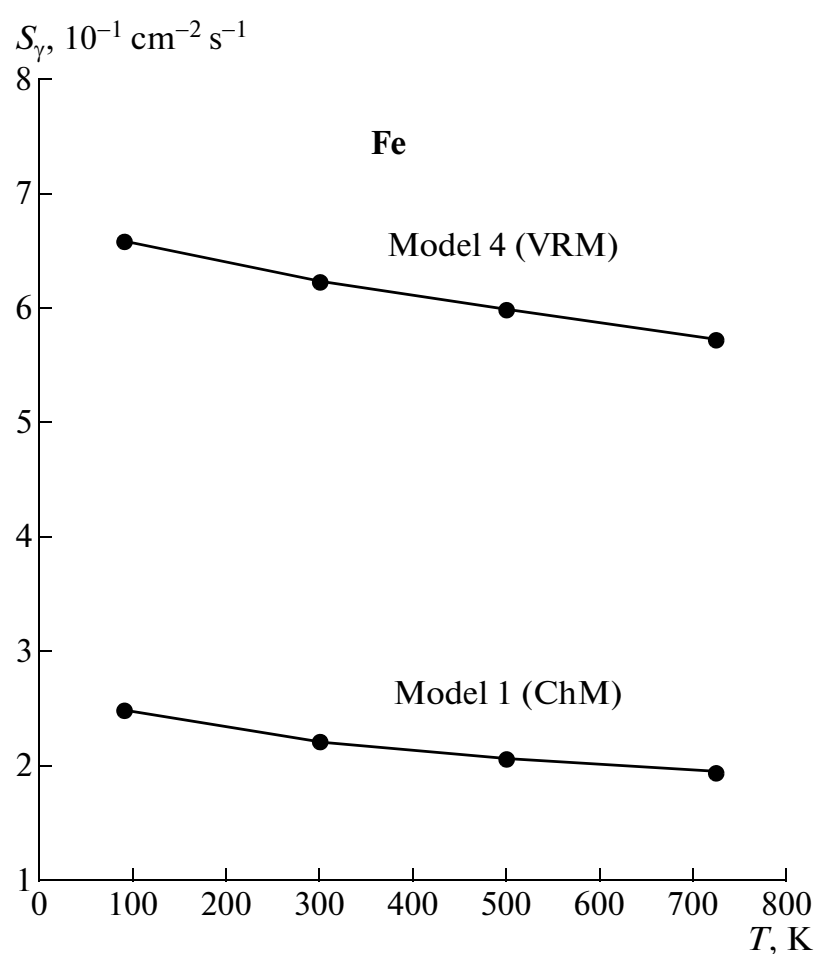

Fig. 8. Same as Fig. 5 for the Fe $7.63+7.65-M e V \gamma$-ray doublet.

capture reactions can be used to estimate the temperature of the subsurface material.

\section{ACKNOWLEDGMENTS}

This work was supported by the Russian Foundation for Basic Research (project code 10-0200327-a). I.G. Mitrofanov thanks the International Space Science Institute in Bern, Switzerland for the possibility of discussing these results in the framework of the "Nuclear Planetology" project and for financial support.

\section{Appendix A}

\section{MODEL FOR THE CALCULATION OF THE NEUTRON FLUXES FROM PLANETARY SURFACES WITH DIFFERENT TEMPERATURES}

The source of primary neutrons at energies of $10^{5}$ to $10^{7} \mathrm{eV}$ is assumed to be isotropic. It is assumed that the source of neutrons with energy $E$ in a layer of surface material at depth $z$ can be described by the product of two independent distribution functions, $f(E) F(z)$. The first factor $f(E)$ describes the energy spectrum of the neutrons:

$$
f(E)=\frac{\alpha E}{E_{0}^{2}+E^{2}} .
$$




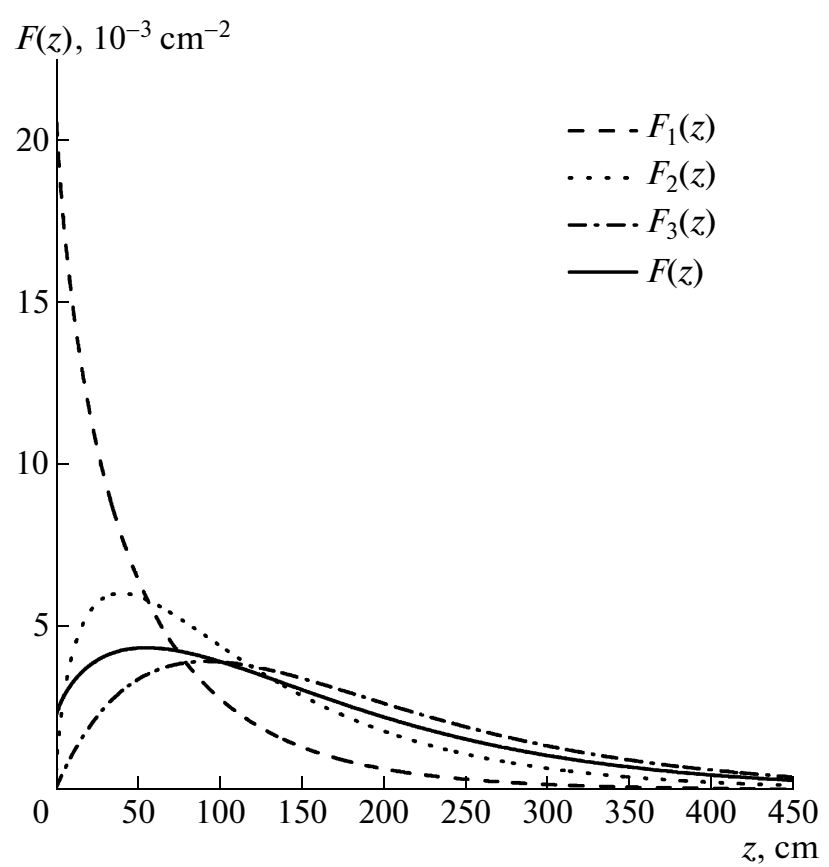

Fig. 9. Dependences of the functions $F_{1}(z), F_{2}(z)$, $F_{3}(z)$, and $F(z)$ on depth $z$ for $l=92.7 \mathrm{~cm}$.

Here, $\alpha$ is a normalization factor, equal to $1 / \ln 10$ for $E_{0}=10^{6} \mathrm{eV}$. The interval of $f(E)$ for energies from $10^{5}$ to $10^{7} \mathrm{eV}$ is equal to unity. This choice of $f(E)$ agrees with experimental data [8, Figs. 275 and 346]. A function of this form phenomenologically describes evaporative and cascade neutrons. Neutrons with energies exceeding $10^{7} \mathrm{eV}$ were not included. The second factor above describes the dependence on the depth, $F(z)$. This function is also normalized to unity and can be represented as a sum of three functions:

$$
\begin{gathered}
F(z)=0.1014 F_{1}(z) \\
+0.2553 F_{2}(z)+0.6433 F_{3}(z), \\
F_{1}(z)=2 \frac{z}{l^{2}} \Gamma\left(-1, \frac{z}{l}\right), \\
F_{2}(z)=\frac{z}{l^{2}} \Gamma\left(0, \frac{z}{l}\right), \\
F_{3}(z)=\frac{z}{l^{2}} \exp \left(-\frac{z}{l}\right),
\end{gathered}
$$

where $\Gamma(\alpha, x) \equiv \int_{x}^{\infty} e^{-t} t^{\alpha-1} d t$ is an incomplete $\Gamma$ function and $l$ is the mean-free path of a primary proton and high-energy cascade nucleons.

The function $F_{1}(z)$ describes the generation of neutrons by cosmic rays (protons with energies of several $\mathrm{GeV}$ ) during single interactions with nuclei of the planetary surface layer. Evaporative and cascade neutrons and first-generation protons arise in this case. The function $F_{2}(z)$ describes the generation of second-generation neutrons during subsequent interactions of the neutrons and first-generation protons with nuclei. The function $F_{3}(z)$ describes the generation of third-generation neutrons. According to our estimates, 16 neutrons are generated by a single primary proton. The numerical coefficients in formula (A.2) correspond to the fact that the neutron multiplication factor for each interaction with a nucleus is 2.52 (for three successive interactions, $2.52^{3}=16$ neutrons are generated).

The number density of element $i$ is determined as

$$
n_{i}=\frac{\rho \alpha_{i}}{1.66 \times 10^{-24} A_{i}},
$$

where $\alpha_{i}$ is the weighted fraction $\left(\sum_{i} \alpha_{i}=1\right)$ and $A_{i}$ is the mass of element $i$ in atomic mass units.

The density of the regolith was taken to be $\rho=$ $1 \mathrm{~g} / \mathrm{cm}^{3}$ (the fluxes of neutrons and $\gamma$-rays do not depend on this quantity). The chemical bonds of the atoms in the regolith were not taken into account. The cross sections for elastic scattering and absorption of neutrons and the angular dependence of the differential elastic cross section were taken from [6].

The cross section for the interaction of a neutron with a nucleus depends on their relative velocity, $\left|\overline{V_{i}}-\overline{V_{n}}\right|$. In all energy intervals, the velocity of the neutron, $\overline{V_{n}}$, was much higher than the velocity of the nucleus $i$ with which it interacts, $\overline{V_{i}}$, i.e., $\mid \overline{V_{i}}-$ $\overline{V_{n}} \mid \approx \overline{V_{n}}$. To take into account the dependence of the elastic-scattering cross section on energy and angle, we applied an expansion of the differential elasticscattering cross section in Legendre polynomials:

$$
\frac{d \sigma}{d \Omega}=\frac{\sigma}{2 \pi} \sum \frac{2 l+1}{2} \alpha_{l} P_{l}(\cos \vartheta),
$$

where $\sigma$ is the total elastic-scattering cross section. The expansion coefficients $\alpha_{l}$ depend on the energy (their number was up to 9), and their values were taken from [6].

The cross section for the interaction of a proton with a nucleus with mass $A_{i}$ with the generation of neutrons is $\sigma_{i}=0.05 A_{i}^{2 / 3}$ barn, and depends only weakly on the energy in the range $0.2-8 \mathrm{GeV}[8$, Figs. 239, 240]. We used this same cross section for the generation of second- and third-generation neutrons. The mean free path is

$$
l=\frac{1}{\sum_{i} n_{i} \sigma_{i}},
$$

where

$$
\begin{gathered}
n_{i}=0.6022 \times 10^{24} \frac{\rho \alpha_{i}}{A_{i}}, \\
l=\frac{33.21}{\sum_{i} \rho \alpha_{i} A_{i}^{-1 / 3}} .
\end{gathered}
$$

ASTRONOMYREPORTS Vol.56 No.4 2012 
For the regolith compositions considered (Table 1) and with $\rho=1 \mathrm{~g} / \mathrm{cm}^{3}$, we obtain the following estimates for the mean free path

$$
\begin{array}{ll}
l=92.7 \mathrm{~cm} & \text { for Model } 1, \\
l=92.0 \mathrm{~cm} & \text { for Model } 2, \\
l=92.7 \mathrm{~cm} & \text { for Model } 3, \\
l=94.0 \mathrm{~cm} & \text { for Model } 4 .
\end{array}
$$

Figure 9 presents the functions $F_{1}(z), F_{2}(z), F_{3}(z)$, and $F(z)$ for $l=92.7 \mathrm{~cm}$. The dependence of the source of neutrons on the depth, $F(z)$, agrees with the results of [10]. In the thermal and epithermal energy ranges, $k T / 10<E<10 \mathrm{eV}$, the neutron spectrum formed as a result of collisions with nuclei in the regolith having a Maxwellian energy distribution. It was assumed that a neutron with mass $m_{1}$, velocity $\bar{v}_{1}$, and energy $E_{1}=m_{1} v_{1}^{2} / 2$ was scattered on a nucleus with mass $m_{2}$ and velocity $\bar{v}_{2}$, with this velocity being random and described by a Maxwellian distribution:

$$
\frac{d \omega}{d v_{2}}=4 \pi\left(\frac{m_{2}}{2 \pi k T}\right)^{\frac{3}{2}} v_{2}^{2} \exp \left(-\frac{m_{2} v_{2}^{2}}{2 k T}\right)
$$

with the velocity $v_{2}$ normalized to unity. The velocity vector was isotropically oriented.

The neutron's velocity after the scattering, $\bar{v}_{1}^{\prime}$, was [9]

$$
\bar{v}_{1}^{\prime}=\frac{m_{2}}{m_{1}+m_{2}} v \bar{n}_{0}+\frac{m_{1} \bar{v}_{1}+m_{2} \bar{v}_{2}}{m_{1}+m_{2}},
$$

where

$$
\begin{gathered}
\bar{v}=\bar{v}_{1}-\bar{v}_{2}, \\
v=\sqrt{v_{1}^{2}+v_{2}^{2}-2 v_{1} v_{2} \bar{n}_{1} \bar{n}_{2}},
\end{gathered}
$$

and the unit vector $\bar{n}_{0}$ defines the direction of the neutron velocity in the center of mass system. It was assumed that this unit vector was isotropically distributed.

The center of mass velocity for the two particles $\bar{v}_{c}$ appears in the second term in (A.7):

$$
\begin{gathered}
\frac{m_{1} v_{1} \bar{n}_{1}+m_{2} v_{2} \bar{n}_{2}}{m_{1}+m_{2}}=v_{\mathrm{c}} \bar{n}_{\mathrm{c}}, \\
v_{\mathrm{c}}=\frac{1}{1+A_{i}} \sqrt{v_{1}^{2}+A_{i}^{2} v_{2}^{2}+2 A_{i} v_{1} v_{2} \bar{n}_{1} \bar{n}_{2}}, \\
\bar{n}_{\mathrm{c}}=\frac{v_{1} \bar{n}_{1}+A_{i} v_{2} \bar{n}_{2}}{\sqrt{v_{1}^{2}+A_{i}^{2} v_{2}^{2}+2 A_{i} v_{1} v_{2} \bar{n}_{1} \bar{n}_{2}}}, \\
A_{i}=\frac{m_{2}}{m_{1}} .
\end{gathered}
$$

The subscript $i$ corresponds to an element making up the composition of the regolith. It follows from
(A.7) and (A.8) that

$$
\begin{gathered}
v_{1}^{\prime}=\frac{1}{1+A_{i}} \\
\times \sqrt{A_{i}^{2} v^{2}+\left(1+A_{i}\right)^{2} v_{c}^{2}+2 A_{i}\left(1+A_{i}\right) v v_{c} \bar{n}_{0} \bar{n}_{c}}, \\
n_{1}^{\prime}=\frac{A_{i} v \bar{n}_{0}+\left(1+A_{i}\right) v_{c} \bar{n}_{c}}{\sqrt{A_{i}^{2} v^{2}+\left(1+A_{i}\right)^{2} v_{c}^{2}+2 A_{i}\left(1+A_{i}\right) v v_{c} \bar{n}_{0} \bar{n}_{c}}} .
\end{gathered}
$$

The directions of the neutron velocities before scattering $\bar{n}_{1}$ and after scattering $\bar{n}_{1}^{\prime}$, and also the vectors $\bar{n}_{0}, \bar{n}_{c}$, and $\bar{n}_{2}$, are determined by the sines and cosines of the corresponding angles $\vartheta$ and $\varphi$ in a spherical coordinate system with its $z$ axis directed normal to the surface. A derivation of the formulas for the sines and cosines of these angles is not presented here for simplicity.

\section{Appendix B}

\section{MODEL FOR CALCULATING THE $\gamma$-RAY FLUXES IN NEUTRON CAPTURE LINES OF Al, Si, Ca, AND Fe GENERATED AT THE SURFACE OF MERCURY}

We present here calculated fluxes in the neutroncapture $\gamma$-ray lines of ${ }^{27} \mathrm{Al}$ at $7.72 \mathrm{MeV},{ }^{28} \mathrm{Si}$ at $3.54 \mathrm{MeV},{ }^{40} \mathrm{Ca}$ at $1.94 \mathrm{MeV}$, and ${ }^{56} \mathrm{Fe}$ at $(7.63+$ 7.65) $\mathrm{MeV}$. The $7.72 \mathrm{MeV} \gamma$-ray line has a production efficiency of 0.30 ; in other words, for each neutron captured by a ${ }^{27} \mathrm{Al}$ nucleus, on average, $0.30 \gamma$-rays in this line are generated [6]. The $3.54-\mathrm{MeV}$ line of $\mathrm{Si}$ has a production efficiency of 0.70 . We assumed a normal isotopic composition for the crust, with $92.23 \%$ of the Si being ${ }^{28} \mathrm{Si}$, so that the overall production efficiency for $\gamma$-rays in this line was 0.667 . The production efficiency of the ${ }^{40} \mathrm{Ca} 1.94 \mathrm{MeV} \gamma$-ray line was 0.83; for a normal isotopic regolith composition with $96.9 \%$ of $\mathrm{Ca}$ being ${ }^{40} \mathrm{Ca}$, this production efficiency becomes 0.80 . The production efficiencies for the ${ }^{56} \mathrm{Fe} 7.63 \mathrm{MeV}$ and $7.65 \mathrm{MeV} \gamma$-ray lines are 0.29 and 0.25 , respectively; for a normal isotopic composition with $91.72 \%$ of $\mathrm{Fe}$ being ${ }^{56} \mathrm{Fe}$, these production efficiencies become 0.27 and 0.23 , respectively.

We used the neutron spectrum over the entire range of energies from $10 \mathrm{MeV}$ to $k T / 10$ when computing the $\gamma$-ray line fluxes. The main contribution is made by neutrons with low energies, since the neutron capture cross section is proportional to $1 /|\Delta v|$, where $\Delta v$ is the relative velocity between the neutron and capturing nucleus. 
Table 10. Cross section for the creation of electron-positron pairs $\sigma\left(Z, E_{\gamma}\right)$ in barn for the ${ }^{27} \mathrm{Al}(7.7240 \mathrm{MeV}),{ }^{28} \mathrm{Si}$ $(3.5390 \mathrm{MeV}),{ }^{40} \mathrm{Ca}(1.9427 \mathrm{MeV})$, and ${ }^{56} \mathrm{Fe}(7.6312+7.6456 \mathrm{MeV}) \gamma$-ray lines

\begin{tabular}{l|c|c|c|c|c}
\hline \multirow{2}{*}{ Element } & \multicolumn{5}{|c}{$\gamma$-ray line energy MeV } \\
\cline { 2 - 6 } & 1.94 & 3.54 & 7.63 & 7.65 & 7.72 \\
\hline $\mathrm{Fe}$ & 0.1142 & 0.4704 & 1.1602 & 0.1621 & 1.1724 \\
$\mathrm{Cr}$ & 0.0958 & 0.3999 & 0.9924 & 0.9941 & 1.0092 \\
$\mathrm{Ti}$ & 0.0792 & 0.3357 & 0.8375 & 0.8389 & 0.8463 \\
$\mathrm{Ca}$ & 0.0645 & 0.2771 & 0.6457 & 0.6966 & 0.7028 \\
$\mathrm{Si}$ & 0.0302 & 0.1259 & 0.3474 & 0.348 & 0.3511 \\
$\mathrm{Al}$ & 0.0259 & 0.1173 & 0.3008 & 0.3013 & 0.3041 \\
$\mathrm{Mg}$ & 0.0219 & 0.1000 & 0.2576 & 0.2581 & 0.2604 \\
$\mathrm{O}$ & 0.0095 & 0.0451 & 0.1179 & 0.1181 & 0.1192 \\
\hline
\end{tabular}

Table 11. Attenuation coefficients $k_{c}, k_{p}$, and $k_{t}$ for the ${ }^{27} \mathrm{Al}(7.7240 \mathrm{MeV}),{ }^{28} \mathrm{Si}(3.5390 \mathrm{MeV}),{ }^{40} \mathrm{Ca}(1.9427 \mathrm{MeV})$, and ${ }^{56} \mathrm{Fe}(7.6312+7.6456 \mathrm{MeV}) \gamma$-ray lines for regolith models ChM, ECM, RRM, and VRM

\begin{tabular}{c|c|c|c|c|c}
\hline $\begin{array}{c}\gamma \text {-ray line, } \\
\text { MeV }\end{array}$ & $\begin{array}{c}\text { Attenuation } \\
\text { coefficient }\end{array}$ & $\begin{array}{c}\text { Model 1 } \\
(\text { ChM })\end{array}$ & $\begin{array}{c}\text { Model 2 } \\
(\text { ECM) }\end{array}$ & $\begin{array}{c}\text { Model 3 } \\
\text { (RRM) }\end{array}$ & $\begin{array}{c}\text { Model 4 } \\
\text { (VRM) }\end{array}$ \\
\hline \multirow{2}{*}{1.94} & $k_{c}$ & $4.43 \times 10^{-2}$ & $4.43 \times 10^{-2}$ & $4.43 \times 10^{-2}$ & $4.42 \times 10^{-2}$ \\
& $k_{p}$ & $5.34 \times 10^{-4}$ & $5.34 \times 10^{-4}$ & $5.29 \times 10^{-4}$ & $5.75 \times 10^{-4}$ \\
& $k_{t}$ & $4.48 \times 10^{-2}$ & $4.48 \times 10^{-2}$ & $4.48 \times 10^{-2}$ & $4.48 \times 10^{-2}$ \\
3.54 & $k_{c}$ & $3.10 \times 10^{-2}$ & $3.11 \times 10^{-2}$ & $3.11 \times 10^{-2}$ & $3.09 \times 10^{-2}$ \\
& $k_{p}$ & $2.42 \times 10^{-3}$ & $2.33 \times 10^{-3}$ & $2.40 \times 10^{-3}$ & $2.57 \times 10^{-3}$ \\
& $k_{t}$ & $3.34 \times 10^{-2}$ & $3.34 \times 10^{-2}$ & $3.35 \times 10^{-2}$ & $3.35 \times 10^{-2}$ \\
7.63 & $k_{c}$ & $1.85 \times 10^{-2}$ & $1.85 \times 10^{-2}$ & $1.85 \times 10^{-2}$ & $1.84 \times 10^{-2}$ \\
& $k_{p}$ & $6.20 \times 10^{-3}$ & $5.99 \times 10^{-3}$ & $6.18 \times 10^{-3}$ & $6.57 \times 10^{-3}$ \\
7.64 & $k_{t}$ & $2.47 \times 10^{-2}$ & $2.45 \times 10^{-2}$ & $2.47 \times 10^{-2}$ & $2.50 \times 10^{-2}$ \\
& $k_{c}$ & $1.84 \times 10^{-2}$ & $1.85 \times 10^{-2}$ & $1.85 \times 10^{-2}$ & $1.84 \times 10^{-2}$ \\
7.72 & $k_{p}$ & $6.21 \times 10^{-3}$ & $6.00 \times 10^{-3}$ & $6.19 \times 10^{-3}$ & $6.58 \times 10^{-3}$ \\
& $k_{t}$ & $2.46 \times 10^{-2}$ & $2.45 \times 10^{-2}$ & $2.47 \times 10^{-2}$ & $2.50 \times 10^{-2}$ \\
& $k_{c}$ & $1.83 \times 10^{-2}$ & $1.84 \times 10^{-2}$ & $1.84 \times 10^{-2}$ & $1.82 \times 10^{-2}$ \\
& $k_{p}$ & $6.27 \times 10^{-3}$ & $6.06 \times 10^{-3}$ & $6.24 \times 10^{-3}$ & $6.64 \times 10^{-3}$ \\
& $k_{t}$ & $2.46 \times 10^{-2}$ & $2.45 \times 10^{-2}$ & $2.46 \times 10^{-2}$ & $2.48 \times 10^{-2}$ \\
\hline
\end{tabular}

When $\gamma$-rays propagate in a medium, the main process changing their energies is Compton scattering. Another important process is the absorption of the $\gamma$-rays during the creation of electronpositron pairs. The Compton scattering cross section $\sigma_{c}$ is $1.488 \times 10^{-25}, 1.04 \times 10^{-25}, 0.6193 \times 10^{-25}$, $0.6184 \times 10^{-25}$, and $0.614 \times 10^{-25} \mathrm{~cm}^{2}$ for 1.94 , $3.54,7.63,7.65$, and $7.72 \mathrm{MeV} \gamma$-rays, respectively. Estimates of the minimum electron number density that makes it necessary to take Compton scattering into account differ for the various models (Table 1):

$$
n_{1 e}=2.98 \times 10^{23} \mathrm{~cm}^{-3} \quad \text { for Model } 1,
$$

$$
\begin{array}{ll}
n_{2 e}=2.99 \times 10^{23} \mathrm{~cm}^{-3} & \text { for Model 2, } \\
n_{3 e}=2.99 \times 10^{23} \mathrm{~cm}^{-3} & \text { for Model 3, } \\
n_{4 e}=2.97 \times 10^{23} \mathrm{~cm}^{-3} & \text { for Model 4. }
\end{array}
$$

The attenuation coefficient associated with Compton scattering is $k_{c}=n_{e} \sigma_{c}$. The attenuation coefficient associated with the creation of electronpositron pairs on atoms must be added to this; this coefficient per atom is given by [7]

$$
\begin{gathered}
\sigma\left(Z, E_{\gamma}\right)=Z(Z+1) \\
\times\left(\Phi_{1}(x)+\Phi_{2}(x) Z+\frac{\Phi_{3}(x)}{Z}\right),
\end{gathered}
$$




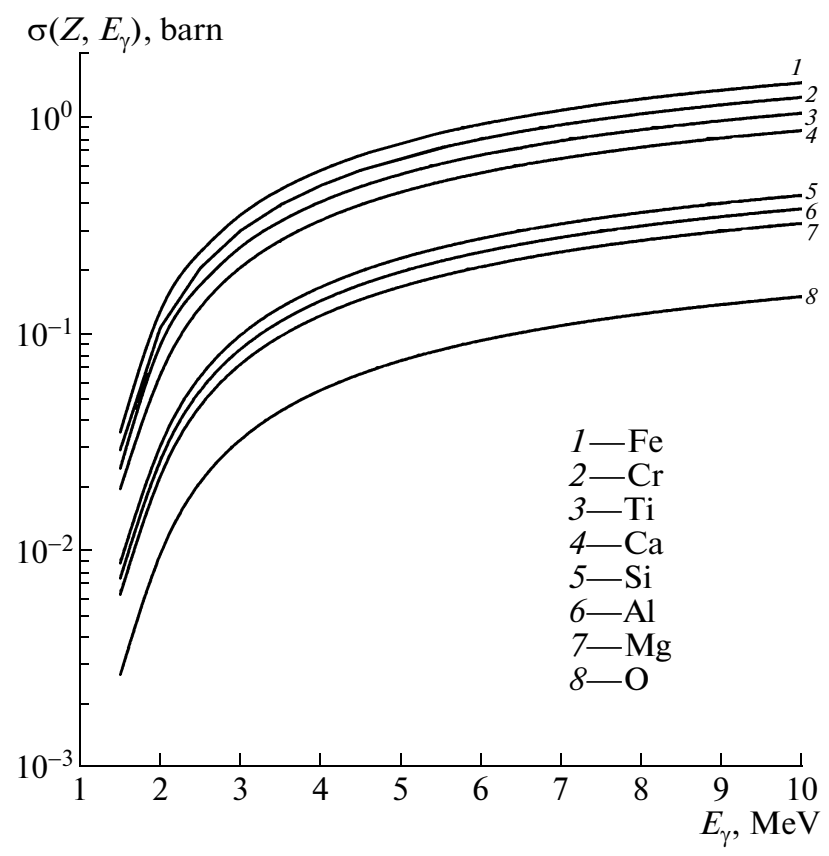

Fig. 10. Dependence of the cross section (per atom) for the creation of electron-positron pairs $\sigma\left(Z, E_{\gamma}\right)$ for various elements on the $\gamma$-ray energy $E_{\gamma}$.

$$
x=\ln \left(\frac{E_{\gamma}}{m_{e} c^{2}}\right),
$$

where $Z$ is the charge of the nucleus. The functions $\Phi_{1}(x), \Phi_{2}(x)$, and $\Phi_{3}(x)$ are specified as polynomials:

$$
\begin{aligned}
& \Phi_{1}(x)=\sum_{n=0}^{5} a_{n} x^{n}, \\
& \Phi_{2}(x)=\sum_{n=0}^{5} b_{n} x^{n}, \\
& \Phi_{3}(x)=\sum_{n=0}^{5} c_{n} x^{n} .
\end{aligned}
$$

This expression for $\sigma\left(Z, E_{\gamma}\right)$ is applicable in the ranges $1 \leq Z \leq 100, E_{\gamma}=1.5 \mathrm{MeV}-100 \mathrm{GeV}$. The dependences of the cross section $\sigma\left(Z, E_{\gamma}\right)$ on the energy $E_{\gamma}$ for the elements considered are shown in Fig. 10.

The corresponding $\gamma$-ray attenuation coefficient $k_{p}$ was calculated using the formula

$$
k_{p}=\sum_{i} n_{i} \sigma\left(Z_{i}, E_{\gamma}\right),
$$

where $Z_{i}$ is the number of electrons in an atom of element $i$ and $n_{i}$ is the number density of element $i$. Table 10 presents the cross sections for the creation of electron-positron pairs per atom $\sigma\left(Z, E_{\gamma}\right)$ in barn for the $\gamma$-ray lines considered.

Table 11 presents the attenuation coefficients $k_{c}$ and $k_{p}$, together with the total attenuation coefficient $k_{t}\left[\mathrm{~cm}^{-1}\right]$ for $\rho=1 \mathrm{~g} / \mathrm{cm}^{3}$ for the $1.94,3.54,7.63$, 7.65, and 7.24 MeV $\gamma$-ray lines. During their propagation from the place they are created in the regolith to their escape at the surface, the $\gamma$-rays are attenuated according to the exponential law $\exp \left(-k_{t} z / \mu\right)$, where $z>0$ is the depth at which the $\gamma$-rays are generated, $\mu=\cos \vartheta$, and $\vartheta$ is the angle between the direction of propagation of the $\gamma$-rays and the outward normal to the planetary surface.

\section{REFERENCES}

1. I. Mitrofanov, A. Kozyrev, A. Konovalov, et al., Planet. Space Sci. 58, 116 (2010).

2. J. Morgan and E. Anders, Proc. Natl. Acad. Sci. 77, 6973 (1980).

3. Basaltic Volcanism Study Project: Basaltic Volcanism on the Terrestrial Planets, Geophysical and Cosmochemical Constraints on Properties of Mantles of the Terrestrial Planets (Pergamon Press, New York, 1981$)$, p. 634.

4. B. Fegley and A. G. W. Cameron, Earth Planet. Sci. Lett. 82, 207 (1987).

5. K. A. Goettel, in Mercury, Ed. by F. Vilas, C. R. Chapman, and M. S. Mathew (Univ. Arisona Press, Tucson, Arizona, 1988), p. 613.

6. Evaluated Nuclear Data File (ENDF), http://www-nds.iaea.org/exfor/endf.htm.

7. Physics Reference Manual, http://wwwasd.web.cern.ch/wwwasd/geant4/G4UsersDocuments/UsersGuides/PhysicsReferenceManual/html/index.html.

8. V. S. Barashenkov and V. D. Toneev, Interactions of High-Energy Particles and Nuclei with Nuclei (Atomizdat, Moscow, 1972) [in Russian].

9. L. D. Landau and E. M. Lifshitz, Course of Theoretical Physics, Vol. 1: Mechanics (Nauka, Moscow, 1973; Pergamon Press, New York, 1988).

10. J. Masarik and R. C. Reedy, J. Geophys. Res. 101, 18891 (1996).

Translated by D. Gabuzda 